\title{
Convex games versus clan games*
}

\author{
Rodica Branzei \\ Faculty of Computer Science \\ Alexandru Ioan Cuza University, Romania \\ Dinko Dimitrov \\ Chair of Microeconomics, Faculty of Law and Economics \\ University of Bayreuth, Germany \\ Stef Tijs \\ CentER and Department of Econometrics and Operations Research \\ Tilburg University, The Netherlands
}

\begin{abstract}
In this paper we provide characterizations of convex games and total clan games by using properties of their corresponding marginal games. We show that a "dualize and restrict" procedure transforms total clan games with zero worth for the clan into monotonic convex games. Furthermore, each monotonic convex game generates a total clan game with zero worth for the clan by a "dualize and extend" procedure. These procedures are also useful for relating core elements and elements of the Weber set of the corresponding games.
\end{abstract}

JEL Classification: C71.

Keywords: convex games, core, dual games, marginal games, total clan games, Weber set.

*Financial support from the Alexander von Humboldt Foundation (D. Dimitrov) is gratefully acknowledged. 


\section{Introduction}

The class of convex games was introduced by Shapley (1971) and has attracted a lot of attention because the games in this class have many useful applications. Moreover, solution concepts on this class of games have very appealing properties; we mention here that the core of a convex game is the unique stable set and its extreme points can be easily described, the Shapley value of a convex game is in the barycenter of the core in the sense that it is the average of the marginal vectors. Convex games are in fact totally convex since each subgame of a convex game is also convex. Many equivalent characterizations of this class of games can be found in the cooperative game theory literature. For example, the supermodularity of the characteristic function, the increasing marginal return properties for individual players and for groups of players, and characterizations that deal with the relation between the core and the Weber set (cf. Shapley (1971), Ichiishi (1981), Curiel and Tijs (1991), Curiel (1997)); a characterization of a convex game using the exactness of its subgames can be found in the work of Biswas et al. (1999) and Azrieli and Lehrer (2005).

The class of clan games was introduced by Potters et al. (1989) to model social interaction between a "powerful" group of players (the clan) and "powerless" players (non-clan members). Economic applications of such games include bankruptcy problems, production economies, information acquisition and holding situations (cf. Muto et al. (1988), Potters et al. (1989), Branzei et al. (2001), Tijs et al. (2005)). In the work of Voorneveld et al. (2002) total clan games were introduced as monotonic clan games whose subgames inherit the structure of the original (clan) game.

It is worth mentioning that both convex games and total clan games 
are totally balanced and possess monotonic allocation schemes; specifically, convex games have population monotonic allocation schemes (cf. Sprumont (1990)) and total clan games have bi-monotonic allocation schemes (cf. Branzei et al. (2001) and Voorneveld et al. (2002)). For other properties of (solution concepts on) these two classes of games the reader is referred to Branzei et al. (2005).

In this paper we identify other common features of these classes of games. We start in Section 3 with the characterization of each game in the corresponding class by means of certain properties of appropriately defined marginal games. Further, recent work by Branzei et al. (2006b) on auction games and ring games arising from one-object auction situations has inspired us to study the duality between general convex games and total clan games; this duality relation is presented in Section 4. Specifically, starting with a total clan game with zero worth for the clan we make use of its dual game and restrict it to the non-clan members in order to induce a monotonic convex game. Conversely, we can start with a monotonic convex game, use its dual game and assign zero worth to each coalition not containing a certain group of players as to reach a total clan game with zero worth for the clan. Finally, as we show in Section 5, the way in which the corresponding games are constructed ("dualize and restrict" versus "dualize and extend") is also useful for providing relations between core elements and elements of the Weber set of the corresponding games. Here we show that a specific subset of the Weber set of a total clan game with zero worth for the clan coincides, when restricted to the non-clan members, with the core of the monotonic convex game generated by the "dualize and restrict" procedure. On the other hand, we can reach this specific subset of the Weber set of a total clan game with zero worth for the clan by taking all marginal contribution vectors of 
a monotonic convex game and extending them in an appropriate way. We conclude in Section 6 with some final remarks.

\section{Preliminaries}

A cooperative game with transferable utility (a TU-game) is a pair $(N, v)$, where $N=\{1, \ldots, n\}$ is a set of players and $v: 2^{N} \rightarrow \mathbb{R}$ is a characteristic function satisfying $v(\emptyset)=0$. For any coalition $S \subseteq N, v(S)$ is the worth of coalition $S$, i.e., the members of $S$ can obtain a total payoff of $v(S)$ by agreeing to cooperate. The subgame $\left(S, v_{\mid S}\right)$ is obtained from $(N, v)$ by restricting attention to $S$, i.e., $v_{\mid S}(T)=v(T)$ for all $T \in 2^{S}$. For each coalition $S \in 2^{N} \backslash\{\emptyset\}$ and each player $i \in S$, define

$$
M_{i}(S, v):=v(S)-v(S \backslash\{i\})
$$

to be the marginal contribution of player $i$ to coalition $S$.

Let $\Pi(N)$ be the set of all permutations $\pi: N \rightarrow N$ of $N$, and $P^{\pi}(i)=$ $\left\{q \in N: \pi^{-1}(q)<\pi^{-1}(i)\right\}$ be the set of all predecessors of $i$ with respect to the permutation $\pi$. The marginal contribution vector $m^{\pi}(N, v) \in \mathbb{R}^{N}$ with respect to $\pi$ and $(N, v)$ has the $i$-th coordinate

$$
m_{i}^{\pi}(N, v)=v\left(P^{\pi}(i) \cup\{i\}\right)-v\left(P^{\pi}(i)\right)
$$

for each $i \in N$.

A player $i \in N$ is a veto player in the game $(N, v)$ if $v(S)=0$ whenever

$i \notin S$. A game $(N, v)$ is monotonic if for each $S_{1}, S_{2} \in 2^{N}$ with $S_{1} \subseteq S_{2}$ we have $v\left(S_{1}\right) \leq v\left(S_{2}\right)$. A game $(N, v)$ is called

- superadditive if $v(S \cup T) \geq v(S)+v(T)$ for all $S, T \subseteq N$ with $S \cap T=\emptyset$; 
- convex if $v(S \cup T)+v(S \cap T) \geq v(S)+v(T)$ for all $S, T \subseteq N$.

A game $(N, v)$ is subadditive (concave) if $(N,-v)$ is superadditive (convex). Clearly, each convex (concave) game is also superadditive (subadditive). In what follows we will also use the following alternative characterization of concavity (cf. Curiel (1997)). A game $(N, v)$ is concave if for every pair of coalitions $S_{1}, S_{2} \in 2^{N}$ and every $i \in N$ we have that

$$
i \in S_{1} \subseteq S_{2} \text { implies } M_{i}\left(S_{1}, v\right) \geq M_{i}\left(S_{2}, v\right)
$$

The dual game $\left(N, v^{*}\right)$ of a game $(N, v)$ is defined by $v^{*}(S)=v(N)-$ $v(N \backslash S)$ for all $S \subseteq N$.

An allocation in a game $(N, v)$ is a payoff vector $x \in \mathbb{R}^{n}$. For each $S \subseteq N$, we write $x(S)$ to denote $\sum_{i \in S} x_{i}$. An allocation of $v(N)$ such that this amount is cleared is called efficient, and an allocation $x$ such that $x_{i} \geq v(\{i\})$ for each $i \in N$ is called individually rational. The imputation set of a game $(N, v)$ is the set of all efficient and individually rational allocations, i.e.,

$$
I(N, v)=\left\{x \in \mathbb{R}^{n} \mid x(N)=v(N) \text { and } x_{i} \geq v(\{i\}) \text { for each } i \in N\right\}
$$

Further, an allocation is called stable if any coalition $S \subseteq N$ receives at least its worth $v(S)$. The core of a game $(N, v)$ is the set of all efficient and stable allocations (cf. Gillies (1953)), i.e., the set

$$
\operatorname{Core}(N, v)=\left\{x \in I(v) \mid x(S) \geq v(S) \text { for each } S \in 2^{N}\right\}
$$

Finally, the Weber set $W(N, v)$ of a game $(N, v)$ is the convex hull of the $n$ ! marginal vectors $m^{\pi}(N, v)$, i.e.,

$$
W(N, v)=c o\left\{m^{\pi}(N, v) \mid \pi \in \Pi(N)\right\}
$$




\section{Characterizations via marginal games}

Given a game $(N, v)$ and a coalition $T \subseteq N$, the $T$-marginal game $v^{T}$ : $2^{N \backslash T} \rightarrow \mathbb{R}$ is defined by

$$
v^{T}(S):=v(S \cup T)-v(T)
$$

for each $S \subseteq N \backslash T$.

We focus on characterizations of convex games and of total clan games based on suitably defined marginal games. Marginal games of a convex game have turned out to be useful for proving the fact that the core of a game is a subset of the Weber set (cf. Weber (1988)); they have also played a key role for generating the constrained egalitarian solution for convex games (cf. Dutta and Ray (1989)) and the equal split-off set for arbitrary TU-games (cf. Branzei et al. (2006a)).

It is known that if a game is convex then all its marginal games are also convex (cf. Dutta and Ray (1989)). The next example shows that the superadditivity of a game is not necessarily inherited by its marginal games.

Example 1 Let $N=\{1,2,3\}$ and $v(\{1\})=10, v(\{1,2\})=12, v(\{1,3\})=$ $11, v(\{1,2,3\})=12 \frac{1}{2}$, and $v(S)=0$ for all other $S \subset N$. Clearly, the game $(N, v)$ is superadditive. Its $\{1\}$-marginal game is given by $v^{\{1\}}(\{2\})=$ $v(\{1,2\})-v(\{1\})=2, v^{\{1\}}(\{3\})=11-10=1$, and $v^{\{1\}}(\{2,3\})=2 \frac{1}{2}$. Since $v^{\{1\}}(\{2,3\})=2 \frac{1}{2}<3=v^{\{1\}}(\{2\})+v^{\{1\}}(\{3\})$, the marginal game $\left(\{2,3\}, v^{\{1\}}\right)$ is not superadditive.

As it turns out, the superadditivity of all marginal games of a game $(N, v)$ assures a stronger property than the superadditivity of $(N, v)$, namely the convexity of $(N, v)$. This result has been independently obtained by Branzei et al. (2004) and Martinez-Legaz (2006), and for its proof we refer the reader 
to these papers.

Proposition 1 A game $(N, v)$ is convex if and only if for each $T \in 2^{N}$ the T-marginal game $\left(N \backslash T, v^{T}\right)$ is superadditive.

Remark 1 In view of Proposition 1, a game $(N, v)$ is concave if and only if for each $T \in 2^{N}$ the marginal game $\left(N \backslash T, v^{T}\right)$ is subadditive.

In the sequel we focus on total clan games and their characterization using suitably defined marginal games. The class of clan games was introduced in Potters et al. (1989) to model social interaction between "powerful" players (clan members) and "powerless" players (non-clan members). In a clan game there is a group of "powerful" players with veto power and "powerless" players who operate more profitably in unions than on their own. More precisely, a game $(N, v)$ is a clan game with clan $C \in 2^{N} \backslash\{\emptyset, N\}$ if it satisfies the following four conditions:

(a) $v(S) \geq 0$ for all $S \subseteq N$;

(b) $M_{i}(N, v) \geq 0$ for each $i \in N$;

(c) Clan property: every player $i \in C$ is a veto player, i.e., $v(S)=0$ for each coalition $S$ with $C \nsubseteq S$;

(d) Union property: $v(N)-v(S) \geq \sum_{i \in N \backslash S} M_{i}(N, v)$ if $C \subseteq S$.

For notational convenience, define $\mathcal{P}^{C}:=\{S \subseteq N \mid C \subseteq S\}$ as the collection of all coalitions containing $C$.

A game $(N, v)$ is a total clan game with clan $C \in 2^{N} \backslash\{\emptyset, N\}$ if it is monotonic and $\left(S, v_{\mid S}\right)$ is a clan game (with clan $C$ ) for every $S \in \mathcal{P}^{C}$. Notice that in the definition of a total clan game attention is restricted to coalitions that contain the clan $C$, since the clan property of $(N, v)$ implies that in the other subgames the characteristic function is simply the zero function. Note further that monotonicity implies (a) and (b). As shown by 
Voorneveld et al. (2002), a game $(N, v)$ is a total clan game with clan $C$ if and only if $(N, v)$ is monotonic, every player $i \in C$ is a veto player, and for all coalitions $S_{1}, S_{2} \in \mathcal{P}^{C}$ the following $C$-concavity property holds:

$$
S_{1} \subseteq S_{2} \text { and } i \in S_{1} \backslash C \text { imply } M_{i}\left(S_{1}, v\right) \geq M_{i}\left(S_{2}, v\right) .
$$

Let $N=\{1, \ldots, n\}$ and $C \in 2^{N} \backslash\{\emptyset, N\}$. In what follows we denote by $M V^{N, C}$ the set of all monotonic games on $N$ satisfying the veto player property with respect to each player $i \in C$.

Proposition 2 Let $(N, v) \in M V^{N, C}$. Then $(N, v)$ is a total clan game with clan $C$ if and only if the marginal game $\left(N \backslash C, v^{C}\right)$ is a concave game.

Proof. Let $(N, v) \in M V^{N, C}$ be a total clan game with clan $C$. Then for $i \in S \subseteq T \subseteq N$ we have

$$
\begin{aligned}
v^{C}(S)-v^{C}(S \backslash\{i\}) & =v(C \cup S)-v((C \cup S) \backslash\{i\}) \\
& \geq v(C \cup T)-v((C \cup T) \backslash\{i\}) \\
& =v^{C}(T)-v^{C}(T \backslash\{i\}),
\end{aligned}
$$

where the inequality follows from the $C$-concavity of $(N, v)$. Hence, $(N, v)$ is a concave game.

Suppose now that $\left(N \backslash C, v^{C}\right)$ is a concave game. Let $S_{1}, S_{2} \in \mathcal{P}^{C}$, $S_{1} \subseteq S_{2}$, and $i \in S_{1} \backslash C$. Then

$$
\begin{aligned}
M_{i}\left(S_{1}, v\right) & =v\left(S_{1}\right)-v\left(S_{1} \backslash\{i\}\right) \\
& =v^{C}\left(S_{1} \backslash C\right)-v^{C}\left(\left(S_{1} \backslash C\right) \backslash\{i\}\right) \\
& \geq v^{C}\left(S_{2} \backslash C\right)-v^{C}\left(\left(S_{2} \backslash C\right) \backslash\{i\}\right) \\
& =M_{i}\left(S_{2}, v\right),
\end{aligned}
$$

where the inequality follows from the concavity of $\left(N \backslash C, v^{C}\right)$. Thus, $(N, v)$ is a total clan game with clan $C$. 
Given a game $(N, v) \in M V^{N, C}$ and a coalition $T \in 2^{N \backslash C}$, the $C$-based $T$-marginal game $\left(v^{C}\right)^{T}: 2^{N \backslash T} \rightarrow \mathbb{R}$ is defined by

$$
\left(v^{C}\right)^{T}(S):=v(S \cup T \cup C)-v(T \cup C)
$$

for each $S \subseteq N \backslash T$.

We have then the following result.

Proposition 3 Let $(N, v) \in M V^{N, C}$. Then the following assertions are equivalent:

(a) $(N, v)$ is a total clan game with clan $C$;

(b) $\left(N \backslash C, v^{C}\right)$ is a concave game;

(c) $\left(N \backslash(C \cup T),\left(v^{C}\right)^{T}\right)$ is a subadditive game for each $T \subseteq N \backslash C$;

(d) $\left(N \backslash(C \cup T), v^{C \cup T}\right)$ is a subadditive game for each $T \subseteq N \backslash C$.

Proof. Notice that $(\mathrm{a}) \Longleftrightarrow$ (b) follows from Proposition 2 , and (b) $\Longleftrightarrow$ (c) holds by Remark 1. Finally, (c) $\Longleftrightarrow$ (d) follows easily from the definition of a $C$-based $T$-marginal game.

\section{From clan games to convex games and the way back}

In this section we present a useful relation between total clan games with zero worth for the clan and monotonic convex games, being interested in transformations that work across these two classes of games. As it turns out, we can always construct monotonic convex games from total clan games with zero worth for the clan, and total clan games with zero worth for the clan from monotonic convex games. We call the corresponding transformation procedures "dualize and restrict" and "dualize and extend", respectively. 
Let $N=\{1, \ldots, n\}$ and $C \in 2^{N} \backslash\{\emptyset, N\}$ be fixed. We denote by $G^{N}$ the set of all games on $N$ and by $C L A N_{0}^{N, C}$ the set of all total clan games on $N$ with clan $C$ for which $v(C)=0$ is valid. The set of all games on $N \backslash C$ will be denoted by $G^{N \backslash C}$ and the set of all monotonic convex games on $N \backslash C$ by $M C O N V^{N \backslash C}$.

The "dualize and restrict" operator $D^{r}: C L A N_{0}^{N, C} \rightarrow G^{N \backslash C}$ is defined by

$$
D^{r}(N, v)=(N \backslash C, w) \text { for each }(N, v) \in C L A N_{0}^{N, C},
$$

where $w(S):=v^{*}(S)$ for all $S \subseteq N \backslash C$.

Proposition 3 Let $(N, v) \in C L A N_{0}^{N, C}$. Then $D^{r}(N, v) \in M C O N V^{N \backslash C}$.

Proof. Let $(N \backslash C, w):=D^{r}(N, v)$. To show that $(N \backslash C, w)$ is convex, let $i \in N \backslash C$ and $S_{1} \subseteq S_{2} \subseteq(N \backslash C) \backslash\{i\}$. Then

$$
\begin{aligned}
& w\left(S_{2} \cup\{i\}\right)-w\left(S_{2}\right) \\
= & v(N)-v\left(N \backslash\left(S_{2} \cup\{i\}\right)\right)-v(N)+v\left(N \backslash S_{2}\right) \\
= & v\left(N \backslash S_{2}\right)-v\left(N \backslash\left(S_{2} \cup\{i\}\right)\right) \\
\geq & v\left(N \backslash S_{1}\right)-v\left(N \backslash\left(S_{1} \cup\{i\}\right)\right) \\
= & v(N)-v\left(N \backslash\left(S_{1} \cup\{i\}\right)\right)-v(N)+v\left(N \backslash S_{1}\right) \\
= & w\left(S_{1} \cup\{i\}\right)-w\left(S_{1}\right),
\end{aligned}
$$

where the inequality follows from the $C$-concavity of $(N, v)$.

By the monotonicity property of $(N, v)$ we have that $w\left(S_{1}\right)=v(N)-$ $v\left(N \backslash S_{1}\right) \leq v(N)-v\left(N \backslash S_{2}\right)=w\left(S_{2}\right)$ for $S_{1} \subseteq S_{2} \subseteq N \backslash C$, i.e., the game $(N \backslash C, w)$ is monotonic as well.

The "dualize and extend" operator $D^{e}: M C O N V^{N \backslash C} \rightarrow G^{N}$ is defined by

$$
D^{e}(N \backslash C, w)=(N, v) \text { for each }(N \backslash C, w) \in M C O N V^{N \backslash C} \text {, }
$$


where

$$
v(S)= \begin{cases}0 & \text { if } C \nsubseteq S, \\ w(N \backslash C)-w((N \backslash C) \backslash(S \cap(N \backslash C))) & \text { otherwise, }\end{cases}
$$

for all $S \subseteq N$.

Proposition 4 Let $(N \backslash C, w) \in M C O N V^{N \backslash C}$. Then $D^{e}(N \backslash C, w) \in$ $C L A N_{0}^{N, C}$.

Proof. Notice that, by the definition of $v$, each player $i \in C$ is a veto player in the game $(N, v):=D^{e}(N \backslash C, w)$. To prove that $(N, v)$ is monotonic, let $S \subset N$ and $i \in N \backslash S$.

If $C \nsubseteq S$ and either $i \notin C$, or $i \in C$ is such that $C \nsubseteq S \cup\{i\}$, then $v(S)=0=v(S \cup\{i\})$ follows by the definition of $v$.

If $C \nsubseteq S$ and $i \in C$ is such that $C \subseteq S \cup\{i\}$, then we have

$$
\begin{aligned}
& v(S \cup\{i\}) \\
= & w(N \backslash C)-w((N \backslash C) \backslash((S \cup\{i\}) \cap(N \backslash C))) \\
= & w(N \backslash C)-w((N \backslash C) \backslash(S \cap(N \backslash C))) \\
= & v(S) .
\end{aligned}
$$

If $C \subseteq S$, then

$$
\begin{aligned}
& v(S \cup\{i\}) \\
= & w(N \backslash C)-w((N \backslash C) \backslash((S \cup\{i\}) \cap(N \backslash C))) \\
\geq & w(N \backslash C)-w((N \backslash C) \backslash(S \cap(N \backslash C))) \\
= & v(S)
\end{aligned}
$$

where the inequality follows by the monotonicity of $(N \backslash C, w)$. 
It remains to show that $(N, v)$ is $C$-concave. For this, let $S_{1} \subseteq S_{2} \subseteq N$ and $i \in S_{1} \backslash C$. Then

$$
\begin{aligned}
& v\left(S_{1}\right)-v\left(S_{1} \backslash\{i\}\right) \\
= & w\left((N \backslash C) \backslash\left(\left(S_{1} \backslash\{i\}\right) \cap(N \backslash C)\right)\right)-w\left((N \backslash C) \backslash\left(S_{1} \cap(N \backslash C)\right)\right) \\
\geq & \left.w\left((N \backslash C) \backslash\left(\left(S_{2} \backslash\{i\}\right) \cap(N \backslash C)\right)\right)-w\left((N \backslash C) \backslash\left(S_{2} \cap(N \backslash C)\right)\right)\right) \\
= & v\left(S_{2}\right)-v\left(S_{2} \backslash\{i\}\right),
\end{aligned}
$$

where the inequality follows by the convexity of $(N \backslash C, w)$.

Notice finally that it is straightforward to prove the following result.

Proposition 5 Let $D^{r}$ and $D^{e}$ be the "dualize and restrict" and the "dualize and extend" operators, respectively, as introduced above. Then,

(a) $D^{e} \circ D^{r}$ is the identity map on $C L A N_{0}^{N, C}$, and

(b) $D^{r} \circ D^{e}$ is the identity map on $M C O N V^{N \backslash C}$.

\section{Core versus Weber set}

In this section we use the "dualize and restrict" and the "dualize and extend" procedures to relate core elements and elements of the Weber set of corresponding (total clan and monotonic convex) games.

In order to state our results, we will need some additional notation. Let the player set $N$ and $C \in 2^{N} \backslash\{\emptyset, N\}$ be fixed, and let $\Pi(C)$ and $\Pi(N \backslash C)$ denote the set of all permutations of $C$ and $N \backslash C$, respectively. For each $(\tau, \sigma) \in \Pi(C) \times \Pi(N \backslash C)$, we write $m^{(\tau, \sigma)}(N, v)$ to denote the marginal contribution vector with respect to $(N, v) \in C L A N_{0}^{N, C}$ and to the permutation $(\tau, \sigma)$ of $N$ according to which the set of all predecessors of each non-clan 
member includes the clan. We let

$$
W^{\prime}(N, v):=\operatorname{co}\left\{m^{(\tau, \sigma)}(N, v) \mid(\tau, \sigma) \in \Pi(C) \times \Pi(N \backslash C)\right\}
$$

Finally, let $m^{(\tau, \sigma)}(N, v)_{\mid N \backslash C}$ denote the projection of $m^{(\tau, \sigma)}(N, v)$ on $N \backslash C$ and

$$
W^{\prime}(N, v)_{\mid N \backslash C}:=\operatorname{co}\left\{m^{(\tau, \sigma)}(N, v)_{\mid N \backslash C} \mid(\tau, \sigma) \in \Pi(C) \times \Pi(N \backslash C)\right\} .
$$

We have the following result.

Proposition 6 Let $(N, v) \in C L A N_{0}^{N, C}$. Then Core $\left(D^{r}(N, v)\right)=W^{\prime}(N, v)_{\mid N \backslash C}$. Proof. Let $(N \backslash C, w):=D^{r}(N, v)$. By Proposition 3, $(N \backslash C, w) \in M C O N V^{N \backslash C}$ and, hence, Core $(N \backslash C, w)=W(N \backslash C, w)$. Thus, it is sufficient to prove that $W(N \backslash C, w)=W^{\prime}(N, v)_{\mid N \backslash C}$. For this, we show that $m^{\sigma}(N \backslash C, w)=$ $m^{(\tau, \bar{\sigma})}(N, v)_{\mid N \backslash C}$ for each $\sigma \in \Pi(N \backslash C)$ and any $\tau \in \Pi(C)$, where $m^{\sigma}(N \backslash C, w) \in$ $W(N \backslash C, w), m^{(\tau, \bar{\sigma})}(N, v)_{\mid N \backslash C} \in W^{\prime}(N, v)_{\mid N \backslash C}$, and $\bar{\sigma}$ is the reverse order of $\sigma$.

For each $i \in N \backslash C$ we have

$$
\begin{aligned}
m_{i}^{(\tau, \bar{\sigma})}(N, v)_{\mid N \backslash C} & =v\left(P^{(\tau, \bar{\sigma})}(i) \cup\{i\}\right)-v\left(P^{(\tau, \bar{\sigma})}(i)\right) \\
& =v\left(C \cup P^{\bar{\sigma}}(i) \cup\{i\}\right)-v\left(C \cup P^{\bar{\sigma}}(i)\right) \\
& =v\left(N \backslash P^{\sigma}(i)\right)-v\left(N \backslash\left(P^{\sigma}(i) \cup\{i\}\right)\right) \\
& =v(N)-v\left(N \backslash\left(P^{\sigma}(i) \cup\{i\}\right)\right)-v(N)+v\left(N \backslash P^{\sigma}(i)\right) \\
& =w\left(P^{\sigma}(i) \cup\{i\}\right)-w\left(P^{\sigma}(i)\right) \\
& =m_{i}^{\sigma}(N \backslash C, w) .
\end{aligned}
$$

Thus, the assertion follows.

Let $m^{\sigma}(N \backslash C, w)$ be the marginal contributions vector with respect to $(N \backslash C, w)$ and $\sigma \in \Pi(N \backslash C)$. In what follows, let $x^{m^{\sigma}(N \backslash C, w)} \in \mathbb{R}^{N}$ be 
defined by

$$
x_{i}^{m^{\sigma}(N \backslash C, w)}= \begin{cases}0 & \text { if } i \in C, \\ m_{i}^{\sigma}(N \backslash C, w) & \text { if } i \in N \backslash C,\end{cases}
$$

and let

$$
X^{(N \backslash C, w)}=\operatorname{co}\left\{x^{m^{\sigma}(N \backslash C, w)} \mid \sigma \in \Pi(N \backslash C)\right\} .
$$

We are ready now to present our last result.

Proposition 7 Let $(N \backslash C, w) \in M C O N V^{N \backslash C}$. Then $W^{\prime}\left(D^{e}(N \backslash C, w)\right)=$ $X^{(N \backslash C, w)}$.

Proof. Let $(N, v):=D^{e}(N \backslash C, w)$. Since, by Proposition 4, $(N, v) \in$ $C L A N_{0}^{N, C}$, it is sufficient to show that $x^{m^{\sigma}(N \backslash C, w)}=m^{(\tau, \bar{\sigma})}(N, v)$ for each $\sigma \in \Pi(N \backslash C)$ and any $\tau \in \Pi(C)$, where $\bar{\sigma}$ is the reverse order of $\sigma$. We distinguish two cases:

(a) $i \in N \backslash C$. In view of Proposition $6, m_{i}^{(\tau, \bar{\sigma})}(N, v)=m_{i}^{(\tau, \bar{\sigma})}(N, v)_{\mid N \backslash C}=$ $m_{i}^{\sigma}(N \backslash C, w)=x_{i}^{m^{\sigma}(N \backslash C, w)}$.

(b) $i \in C$. We have

$$
m_{i}^{(\tau, \bar{\sigma})}(N, v)=v\left(P^{(\tau, \bar{\sigma})}(i) \cup\{i\}\right)-v\left(P^{(\tau, \bar{\sigma})}(i)\right)=0=x_{i}^{m^{\sigma}(N \backslash C, w)},
$$

where the second equality follows from $(N, v) \in C L A N_{0}^{N, C}$ and $P^{(\tau, \bar{\sigma})}(i) \cup$ $\{i\} \subseteq C$.

Hence, the assertion follows.

\section{Concluding remarks}

In this paper we have studied properties of and relations between monotonic convex games and total clan games with zero worth for the clan that, in our opinion, are central for these two classes of games. The characterizations of these games in terms of their corresponding marginal games have 
disclosed a deeper duality relation between the corresponding games that we handled by introducing the "dualize and restrict" and the "dualize and extend" operators. Applications of this dualism can be found in the analysis of one-object auction situations (cf. Branzei et al. (2006b)) that give rise to auction games (total clan games with a single clan member) and to ring games (special convex games).

\section{References}

[1] Azrieli, Y. and Lehrer, E. [2005] Concavification and convex games, Working Paper, Tel Aviv University.

[2] Biswas, A. K., Parthasarathy, T., Potters, J. and Voorneveld, M. [1999] Large cores and exactness, Games and Economic Behavior 28, 1-12.

[3] Branzei, R., Dimitrov, D. and Tijs, S. [2004] A new characterization of convex games, CentER Discussion Paper 2004-109, Tilburg University.

[4] Branzei, R., Dimitrov, D. and Tijs, S. [2005] Models in cooperative game theory: crisp, fuzzy and multichoice games, Springer Verlag, Heidelberg.

[5] Branzei, R., Dimitrov, D. and Tijs, S. [2006a] The equal split-off set for cooperative games, Banach Center Publications 71, 39-46.

[6] Branzei, R., Fragnelli, V., Meca, A. and Tijs, S. [2006b] Two classes of cooperative games related to one-object auction situations, CentER Discussion Paper 2006-25, Tilburg University. 
[7] Branzei, R., Tijs, S. and Timmer, J. [2001] Information collecting situations and bi-monotonic allocation schemes, Mathematical Methods of Operations Research 54, 303-313.

[8] Curiel, I. [1997] Cooperative game theory and applications: cooperative games arising from combinatorial optimization problems, Kluwer Academic Publishers, Dordrecht.

[9] Curiel, I. and Tijs, S. [1991] The minimarg and the maximarg operators, Journal of Optimization Theory and Applications 71, 277-287.

[10] Dutta, B. and Ray, D. [1989] A concept of egalitarianism under participation constraints, Econometrica 57, 403-422.

[11] Gillies, D. B. [1953] Some theorems on $n$-person games, Ph.D. Thesis, Princeton University Press, Princeton, New Jersey.

[12] Ichiishi, T. [1981] Super-modularity: applications to convex games and to the greedy algorithm for LP, Journal of Economic Theory 25, 283286.

[13] Martinez-Legaz, J. E. [2006] Some characterizations of convex games, in: A. Seeger (Ed.), Recent advances in optimization, Springer Verlag, Heidelberg, 293-303.

[14] Muto, S., Nakayama, M., Potters, J. and Tijs, S. [1988] On big boss games, Economic Studies Quarterly 39, 303-321.

[15] Potters, J., Poos, R., Tijs, S. and Muto, S. [1989] Clan games, Games and Economic Behavior 1, 275-293. 
[16] Shapley, L. [1971] Cores of convex games, International Journal of Game Theory 1, 11-26.

[17] Sprumont, Y. [1990] Population monotonic allocation schemes for cooperative games with transferable utility, Games and Economic Behavior 2, 378-394.

[18] Tijs, S., Meca, A. and Lopez, M. A. [2005] Benefit sharing in holding situations, European Journal of Operational Research 162, 251-269.

[19] Voorneveld, M., Tijs, S. and Grahn, S. [2002] Monotonic allocation schemes in clan games, Mathematical Methods of Operations Research 56, 439-449.

[20] Weber, R. [1988] Probabilistic values for games, in: A. E. Roth (Ed.), The Shapley value: essays in honour of LS Shapley, Cambridge University Press, Cambridge, 101-119. 